\title{
Scalar Perturbation in a Black Hole localized on a Brane
}

\author{
A. B. Pavan and R. D. B. Fontana \\ Instituto de Física, Universidade de São Paulo \\ C.P. 66318, 05315-970, São Paulo-SP, Brazil
}

(Received on 17 October, 2005)

\begin{abstract}
In this work we study a scalar perturbation in a brane black hole with Schwarzschild behavior, localized on the brane. This black hole solution was derived by Casadio, Fabbri and Mazzacurati with the metric satisfying the condition $R=0$, where $R$ is the four-dimensional scalar curvature.
\end{abstract}

\section{INTRODUCTION}

In the last few years there has been a renewed interest in the description of our world using extra dimensions. Of especial interest is Randall Sundrum's model (RS) in which the standard model fields are confined to a 4-dimensional hypersurface (3-brane) embedded in a 5-dimensional space-time (bulk) where only gravity can propagate.

In RS-II [1] the extra dimension is infinitely extended but "warped" by the presence of a non-vanishing bulk cosmological constant $\Lambda$.

The vacuum field equations in the 5-dimensional bulk are

$$
\mathcal{R}_{\mathrm{v} \mu}=\Lambda g_{\mathrm{v} \mu} \quad(v, \mu=0, \ldots, 4) .
$$

Projecting the above equation on the brane and introducing Gaussian normal coordinates $x^{i}(i=0, \ldots 3)$ and $z(z=0$ on the brane), one obtains the constraints at $z=0$,

$$
\mathcal{R}_{\mathrm{iz}}=0, \quad R=\lambda,
$$

where $R$ is the 4-dimensional Ricci scalar, $\lambda$ is the cosmological constant which, in this case, vanishes when a fine tuning between $\Lambda$ and the brane tension is done. The condition $R=0$ is a weaker requirement than the purely 4-dimensional vacuum equations $R_{i j}=0$ and is equivalent to $R_{i j}=E_{i j}$, where $E_{i j}$ is proportional to the projection of the 5-dimensional Weyl tensor on the brane.

If we consider a spherically symmetric metric on the brane, we have

$$
d s^{2}=-f(r) d t^{2}+h(r) d r^{2}+r^{2} d \theta^{2}+r^{2} \sin (\theta)^{2} d \phi^{2} .
$$

The solution of the equation $R=0$ to this metric with the ansatz $f(r)=\left(1-\frac{2 M}{r}\right)$ will be

$$
h(r)=\frac{\left(1-\frac{3 M}{2 r}\right)}{\left(1-\frac{2 M}{r}\right)\left(1-\frac{M \gamma}{2 r}\right)},
$$

where $\gamma$ is an arbitrary constant.

This metric with a Schwarzschild type behavior was derived by Casadio, Fabbri and Mazzacuratti $[2,4]$.

\section{SINGULARITIES AND HORIZON ANALYSIS}

As we can see in the above line-element, there are problems with the coordinate system at four points, namely $r=\frac{3 M}{2}$, $r=2 M, r=0$ and $r=\frac{M \gamma}{2}$. There is also a problem with the signature of the metric which turns out to be Euclidean in some special cases: depending on the value of $\gamma$ in some regions we have no negative eigenvalue in $\mathbf{g}$ meaning that there is no time and so no physical sense.

Before choosing a convenient coordinate system, we must verify the scalars of the line-element. By calculating the scalars $P=R^{\mu v \delta \alpha} R_{\mu v \delta \alpha}$ and $Q=R^{\mu v} R_{\mu \nu}$ we obtain

$$
P \simeq \frac{F_{1}(r)}{r^{6}(-2 r+3 M)^{4}}, \quad Q \simeq \frac{F_{2}(r)}{r^{4}(-2 r+3 M)^{4}} .
$$

Thus, we can see that only two of the four points in the lineelement represent possible singularities in space-time. This is so because the scalars go to infinity when we take the limit $r \rightarrow 0$ and $r \rightarrow \frac{3 M}{2}$, i. e., there are real singularities in $r=0$ and $r=\frac{3 M}{2}$ and in the points $r=2 M$ and $r=\frac{\gamma M}{2}$ we have probably no real singularities. In the Penrose diagram however, the space-time 'ends' in the spacelike singularity at $r=\frac{3 M}{2}$, wich means that the spacelike singularity in $r=0$ does not exist in this Universe.

There is a especial regime for $\gamma=3$ in which, we recover the Schwarzschild space-time, $P=\frac{48 M^{2}}{r^{6}}$ and $Q=0$.

Finally, we are going to show that there is only one event horizont in $r=2 M$ by taking null-radial geodesics for which $\dot{\theta}=0$, and $\dot{\phi}=0[3]$

$$
-h(r) \dot{t}^{2}+f(r) \dot{r}^{2}=0 .
$$

With this equation, we can define the so-called tortoise coordinate, generating the null-coordinates $u$ and $v$ :

$$
d r_{*}=\sqrt{\frac{f(r)}{h(r)}} d r=\sqrt{\frac{(2 r-3 M) r^{2}}{2(r-2 M)^{2}(r-M a)}} d r .
$$

Analysing the behavior of the integral when $r \rightarrow 2 M$, we obtain

$$
r_{*} \rightarrow-\infty,
$$

defining

$$
\begin{aligned}
d u & =d r_{*}+d t, \\
d v & =d r_{*}-d t,
\end{aligned}
$$

as the null-coordinate, we can see in the Penrose Diagram defined by $U=\tanh u$ and $V=\tanh v$ that there are no problems in $r=2 M$ (as stated above) and this is a null surface that is actually the only horizon of the metric. 


\section{SCALAR PERTURBATION IN A SCHWARZSCHILD-CFM BLACK HOLE ON THE BRANE}

We investigate the evolution of a massless scalar field $\Psi(r, t, \theta, \phi)$ on the spacetime. The scalar perturbation is given by $4-D$ Klein-Gordon equation

$$
\frac{1}{\sqrt{g}} \partial_{i}\left(\sqrt{g} g^{i j} \partial_{j} \Psi(r, t, \theta, \phi)\right)=0
$$

with $g=-\operatorname{det}\left(g_{i j}\right)$.

The root square of the determinant of the metric is given by

$$
\sqrt{g}=\sqrt{\frac{(2 r-3 M)}{(2 r-M \gamma)}} r^{2} \sin (\theta) .
$$

Substituting the components of the metric and equation (12) in the Klein-Gordon equation and using the variable separation $\Psi(r, t, \theta, \phi)=\sum_{l, m} R(r, t) Y_{l m}(\theta, \phi)$ we have two decoupled equations, and the radial equation is

$$
\begin{gathered}
{\left[\frac{3 r-6 M}{r(2 r-3 M)}+\frac{1}{2 r-M \gamma}+\frac{2 M}{r(r-2 M)}\right] \frac{g^{11}}{R} \frac{\partial R}{\partial r}+} \\
-\left(\frac{r}{r-2 M}\right) \frac{1}{R} \frac{\partial^{2} R}{\partial t^{2}}+\frac{g^{11}}{R} \frac{\partial^{2} R}{\partial r^{2}}=\frac{l(l+1)}{r^{2}} .
\end{gathered}
$$

Changing to the tortoise coordinate

$$
\begin{gathered}
r=r\left(r_{*}\right) \Longrightarrow \frac{d}{d r_{*}}=\sqrt{\frac{f(r)}{h(r)}} \frac{d}{d r}, \\
\frac{d}{d r_{*}}=\frac{(r-2 M)}{r} \sqrt{\frac{2 r-M \gamma}{2 r-3 M}} \frac{d}{d r},
\end{gathered}
$$

and substituting

$$
R(r, t)=\frac{\psi(r, t)}{r}
$$

we find the equation

$$
-\frac{\partial^{2} \psi}{\partial t^{2}}+\frac{\partial^{2} \psi}{\partial r_{*}^{2}}-V\left(r_{*}\right) \psi=0
$$

where the potential is given by

$$
\begin{gathered}
V\left(r_{*}\right)=f(r) \quad\left(M\left[\frac{r^{2}(5+\gamma)-6 M r(\gamma+1)+6 M^{2} \gamma}{r^{3}(2 r-3 M)^{2}}\right]+\right. \\
\left.+\frac{l(l+1)}{r^{2}}\right) .
\end{gathered}
$$

\section{NUMERIC RESULTS AND DISCUSSIONS}

The solution of equation (17) was obtained by numerical integration [5], where the scalar field $\Psi$ is given as a function of the null coordinate $v=t-r_{*}$. In the figure below we have the evolution of the massless scalar field taking $\gamma=2.0$ and $\gamma=3.5$ and with two values of $l$.

Evolution of the massless scalar field

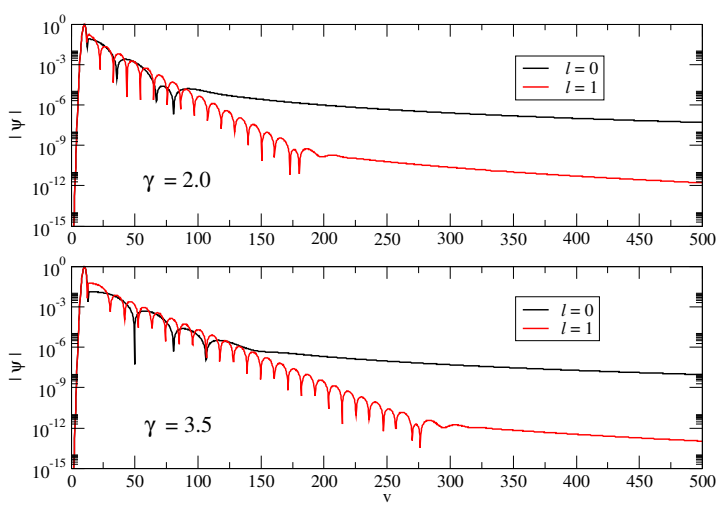

FIG. 1: Field decay in CFM black hole, with $1=0,1$ and two diferent values of $\gamma$

On the above figures we can see that the behavior of the tails and transient is the same as Schwarzschild behavior, i.e., although the potential has an additional term which depends on $\gamma$, it doesn't affect the assintotic behavior.

A more complete analysis about this theme is still in progress [6].

\section{Acknowledgements}

We wish to thank Carlos Molina Mendes for helping with the numerical calculations and Elcio Abdalla for many useful discussions. This work is supported by FAPESP.
[1] L. Randall, R. S. Sundrum, hep-th/9906064.

[2] R. Casadio, A. Fabbri, and L. Mazacurati, gr-qc/011072 v2.

[3] S. W. Hawking, G. F. Ellis, "The large scale structure of space-time”, Cambridge University Press, 1995

[4] R. Casadio, L. Mazacurati, gr-qc/0205129 v2
[5] C. Molina, E. Abdalla, D. Giugno, and A. Saa, Phys. Rev D 69, 10413, 2004.

[6] E. Abdalla, C. Molina, B. Cuadros-Melgar, and A. B. Pavan, in preparation. 